\title{
Fluid Structure Interaction of a loaded Darrieus Marine Current Turbine
}

\author{
M. Belhache ${ }^{1}$, S. Guillou ${ }^{1}$, P. Grangeret ${ }^{1}$, A. Santa-Cruz ${ }^{1}$, F. Bellanger ${ }^{2}$ \\ ${ }^{1}$ Normandy University, University of Caen, Laboratoire Universitaire des Sciences Appliquées de Cherbourg \\ Rue Louis Aragon, BP 87 \\ 50100 Cherbourg-Octeville (France)
}

Phone/Fax number:+0033 233014137, e-mail: mathilde.belhache@unicaen.fr, sylvain.guillou@unicaen.fr

${ }^{2}$ Normandy University, University of Caen, IUT de Cherbourg-Octeville,

Rue Max-Pol Fouchet BP 82,

50130 Cherbourg-Octeville

\begin{abstract}
The present study aims to develop a model to simulate in 2 dimensions, the motion of the fluid and of the loaded Darrieus turbine without imposing the rotational speed. A resistive torque has been added to account for the load of a generator. Experimental and numerical studies were conducted and presented here. The experiment was set-up in the air first, on a wind turbine for reason of convenience. The simulations were made with Fluent (v14) for which a User Defined Function was developed. Then the experimental conditions were reproduced numerically for three cases. The study focuses on the determination of a factor $(f)$ giving the relationship between the experimental and the simulated resistive torque in 2 dimensions. The results are quite satisfying in this preliminary study as they demonstrate that the model developed works well. Some improvement has to be made to refine the value of $f$.
\end{abstract}

\section{Key words}

Vertical Axis Marine Turbine, Fluid Structure Interaction, Power Conversion System, Numerical Simulation, Wind Tunnel experiment.

\section{Introduction}

\section{A. Context}

Owing to the exhaustion of classic energy resources (fossil fuels) exploited since the industrial revolution as well as the potential threat of climate change, the issue of energy supply is the challenge of the XXI century. Since the Earth summit in Rio de Janeiro in 1992, there is a consensus in the need to develop renewable energies. Renewable energies exit in various forms and some expressions can be found in the ocean. The most mature marine energy conversion system is offshore wind turbines and the next systems on the way towards commercialization are marine turbines. Harvesting tidal energy has the advantage to be predictable by opposition to the intermittency of wind energy.
Regarding marine turbine, no standard has emerged yet. If the horizontal axis has been favored for the most advanced prototypes as it could benefit from the experience and years of development invested in wind energy, the vertical axis type, patented in 1926 in France, undergo renewed interest. Indeed the vertical axis turbine presents some characteristics that are interesting for naturel flow. First, the turbine can accept flow from any direction which is convenient on account of flow reversal, besides high potential site may present rotary current [1]. Moreover, in the marine environment flows are turbulent by nature and recent studies tend to demonstrate that the performances of vertical axis turbine are not altered by turbulent flows but on the contrary can be increased [2]. The present study focuses on the numerical prediction of the behavior of a vertical axis marine turbine connected to a generator.

\section{B. Darrieus characterisation : numerical and experimental modeling strategies}

Numerical studies of marine and wind turbines always follow the same pattern: for a given fluid velocity, several rotational speeds of the engine are tested in order to build the non-dimensional power coefficient curve $(\mathrm{Cp}-\lambda$ curve) that pictures the power coefficient $(\mathrm{Cp})$ against the tip-speed ratio $\left(\lambda=\dot{\theta} / \mathrm{U}_{0}\right)$ for a specific fluid velocity $\left(\mathrm{U}_{0}\right)$. The rotational speed $(\dot{\theta})$ is then forced and remains constant over the whole revolution of the turbine ([3]-[5]). The curve enables the identification of the point of maximum performance. The working condition of the turbine for the fluid velocity considered is then supposed to be somewhere on the curve depending on the load applied.

Experimental works ([6]-[9]) apply the same procedure. In all cases a generator is used to drive the turbine at a desired rotation speed. Some questions arise from this method: does forcing numerically and experimentally the rotational speed are the right approaches? Are the forces and moments (intensity and oscillations) well predicted? 
Is the rotational speed constant during a rotation? Thus the objective of this study is to simulate the overall system behavior with a real interaction between the fluid, the turbine, and the load of a generator in order to better predict:

- The forces acting on the rotor for structural analysis

- The turbine behavior

- The power extracted and power quality

In this preliminary work, numerical and experimental results will be correlated, first, on a wind turbine. Note that the final goal is to apply the method to a marine turbine. A vertical axis marine current turbine prototype has already been realized, it is optimized to have it driven by the fluid without any external help (Figure 1).

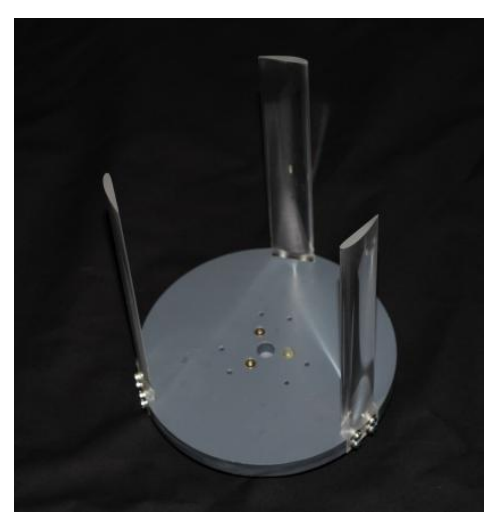

Fig 1. Vertical axis marine current turbine prototype for water channel test.

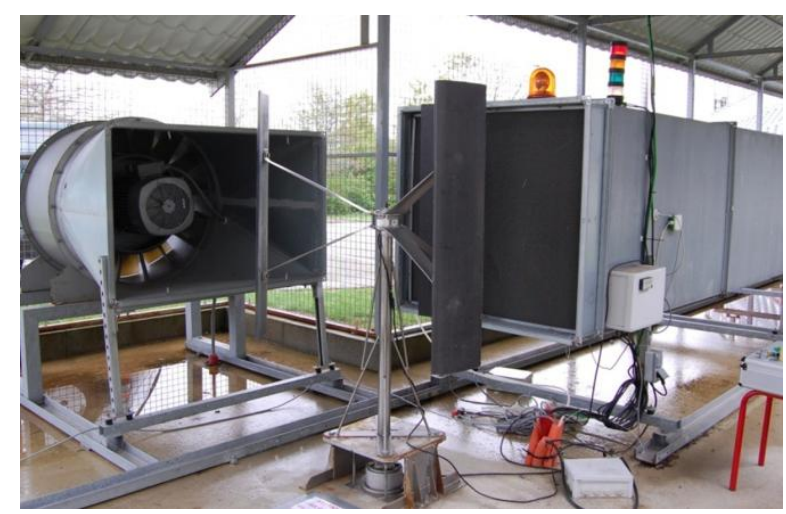

Fig 2. Experimental wind tunnel

\section{Experimental Set-Up}

With a concern of speed and efficiency some preliminary tests were performed in an open wind tunnel on a threebladed Darrieus wind turbine (fig. 2). The experimental work has been favored in air first as it is easier to have the turbine working in free conditions with higher Reynolds number. Experiment in the fluid water brings more constraints; owing to water density the maximum velocity in the channel is reduced. As a matter of fact the size of the prototype is limited. Thus the prototype operates at lower Reynolds numbers and the side effects make it more difficult to have the turbine forced by the fluid.

\section{A. Turbine characteristics}

The turbine is a three-bladed vertical axis turbine of 1 meter diameter (D). Blades are straight, 1 meter high and show a curved profile with a chord length of $0.21 \mathrm{~m}$ (c). The core of the blade is made of acetal resin covered with an external envelope of Carbon (fig 3). The rotating shaft extends to the hub situated at rotor mid-height. Each blade is attached to the hub with two aluminium rigid arms that form a trapezoid. The generator installed is a reversible permanent magnet synchronous motor for electrically assisted cycles

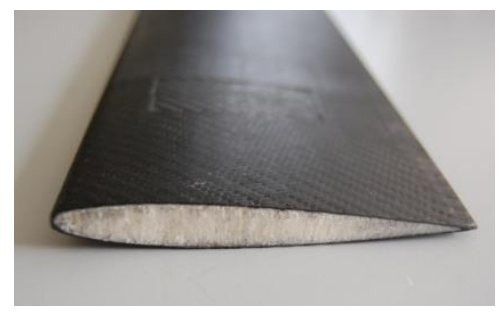

Fig 3. Example of the cross section of a NACA0012 blade builds with the same material.

\section{B. Synchronous generator characterization}

The efficiency of a generator is not constant but varies with the level of torque transmitted to the system and with the rotational velocity owing to iron and mechanical losses. So the first step was to characterize the generator according to the mechanical power developed and the angular velocity of the turbine. The generator was evaluated on a test rig where it was driven by a motor shaft at constant speed. The motor rotated at a constant velocity and supplied a mechanical power $P_{m}$, and the rotation of the generator produced an electrical power $P_{e}$. The efficiency was thus determined as the ratio of $P_{e}$ to $P_{m}$. The performance curves of the generator according to the mechanical power for various rotational speeds are given on fig 4 .

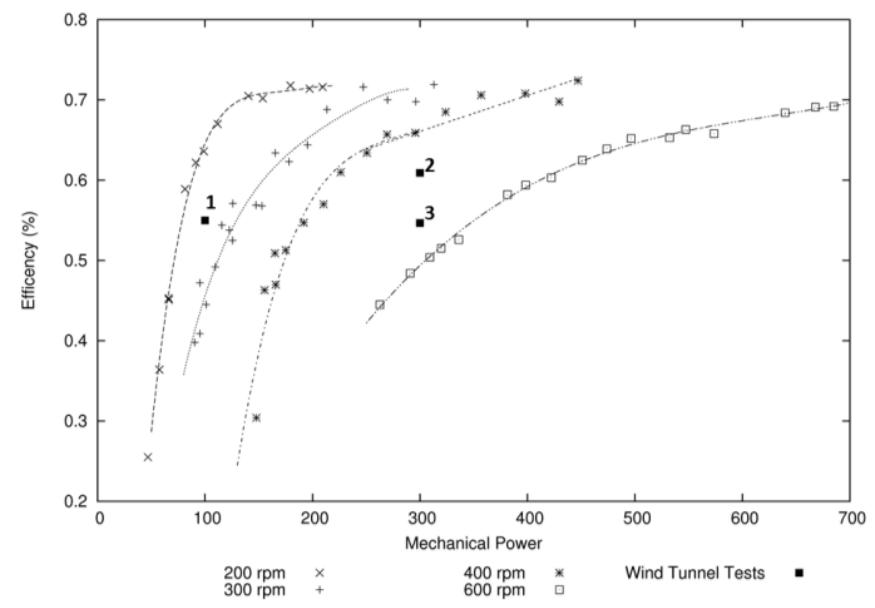

Fig 4. Generator performance behavior in relation to the mechanical power for different rotational speeds. The points represent experimental data and the curves are fitted laws. 


\section{Wind Tunnel Experiment}

The experimental test took place in an open wind tunnel (fig.2) of $1 \mathrm{~m} \mathrm{x} 1 \mathrm{~m}$ in section. The tunnel is $12 \mathrm{~m}$ long equipped with honeycomb in order to laminerize the flow. The wind turbine was positioned on a base so that its axis was $0.6 \mathrm{~m}$ from the tunnel outlet. The objective of the tests was to have different couple $U_{0} / P_{m}$ corresponding to different generator efficiencies $(\eta)$ with the following target values for the mechanical power: 100, 300 and $500 \mathrm{~W}$. For each test, a specific air velocity at the outlet of the tunnel is asked to the command system. The rotational velocity $(\dot{\theta})$ and the electrical power produced $(P e)$ were measured. The mechanical power $\left(P_{m}\right)$ developed by the turbine is deduced from the two latter values and the generator characterization curves. A dimmer was then used to accelerate or slow down the turbine to have the duet $\dot{\theta} / P e$ matching with the target mechanical power. Table I summarises the experimental test conditions. The flow velocity at the outlet of the wind tunnel was not recorded and the level of turbulence is unknown.

Table I. - Experimental test conditions

\begin{tabular}{|c|c|c|c|c|c|}
\hline Test & $\begin{array}{c}\boldsymbol{U}_{\boldsymbol{0}} \\
(\mathbf{m} / \mathbf{s})\end{array}$ & $\begin{array}{c}\dot{\boldsymbol{\theta}} \\
(\mathbf{r a d} / \mathbf{s})\end{array}$ & $\boldsymbol{P e}(\boldsymbol{W})$ & $\boldsymbol{\eta}$ & $\boldsymbol{P}_{\boldsymbol{m}}(\boldsymbol{W})$ \\
\hline $\mathbf{1}$ & 7.13 & 26.3 & 54 & 0.54 & 100 \\
\hline $\mathbf{2}$ & 9.9 & 44.6 & 210 & 0.7 & 300 \\
\hline $\mathbf{3}$ & 10.8 & 55.6 & 210 & 0.7 & 300 \\
\hline
\end{tabular}

\section{Modeling parameters}

\section{A. Domain and Grid Setting}

The domain is 2 dimensional, equivalent to a plan situated mid-height of the turbine. It extends 4D upstream and 14D downstream. The lateral sides are 2.8D distant from the rotation axis, which corresponds to a blockage ratio of 0.18 . The computational domain was divided in two parts, a stationary sub-domain and a rotating sub-domain separated by a sliding interface to enable the rotation of the turbine. The rotating sub-domain is a $3 \mathrm{D}$ circle including the turbine, it's position is updated at each time step. The whole domain was discretized with 503318 elements composed of 13570 elements inside the stationary subdomain and 489750 in the rotating domain. The vertical axis turbine hydrodynamic is very complex, especially in stall regime: attached and separated flow as well as reattachment may occur simultaneously. Moreover the blades can interact with their own wake and the one created by the other elements of the rotor. On account of this unsteady nature, a fine discretization inside the rotor is essential, particularly around the blade. To capture the flow feature inside the boundary layer, a boundary layer cmesh was built around each blade (fig. 5). It develops half of a chord length normally to the blade and is composed of 56113 cells shared out in 120 layers.

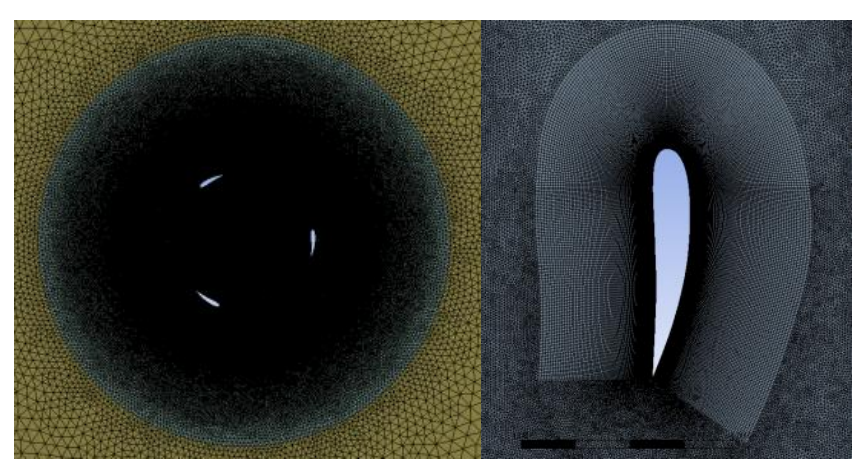

Fig 5. Boundary layer C-mesh around a blade (left) and rotating sub-domain mesh (right)

\section{B. Calculation parameters}

An Unsteady Reynolds Average Navier-Stokes (URANS) approach was chosen for the turbulent modeling. For turbulence closure, the k- $\omega$ SST Model was used owing to its capability to deal with the specific flow features related to the operation of vertical axis turbine (strong adverse pressure gradient and flow reversal) [10]. The velocity-pressure coupling corresponds to the SIMPLEC method and the spatial discretization is of second order. A symmetry condition was applied on the wall domain, the inlet is defined as a constant velocity inlet with $5 \%$ of turbulence intensity and a constant pressure condition was imposed on the outlet. Blades were defined as wall with no slip condition. The time step defined for each test is given in table II, it corresponds to a rotation angle of $0.75^{\circ}$. The relative convergence criterion is set to 0.05 with a maximum of 100 iterations per time step.

For each tests, only the velocity inlet and resistive torque were imposed, all other values were calculated. The velocity ordered to the wind tunnel control console was corrected with 3-d anemometer measurements. Each simulation was initialized with an imposed constant angular velocity case. The angular velocity was chosen close to the target one in order to reduce the computational time. The initialization cases were run on 5 revolutions until the forces and moment reach a periodic behavior. The Reynolds number and tip speed ratio corresponding to each case are given in table I.

\section{Modeling strategy}

In order to simulate the fluid structure interaction of a three-bladed vertical axis marine turbine connected to a generator with a defined resistive torque; a User Defined Function (UDF) has been developed in Ansys Fluent (v.14). At each time step the function gets the calculated force and moment applied on the blade and resolves equation 1 to determine the turbine rotational velocity $(\dot{\theta})$. Once the rotational speed is computed, it is passed on fluent for an update of the mesh position before the next time step. 


$$
\left[3 I_{1}+m r^{2}+I_{2}+\frac{I_{3}}{0.23}\right] \ddot{\theta}=-C r+\sum_{i=1}^{3} \overrightarrow{M_{f_{l / 0}}} \cdot \vec{z}
$$

$$
\operatorname{Cr}=\boldsymbol{f} \cdot \frac{P_{m}}{\dot{\theta}_{m}}
$$

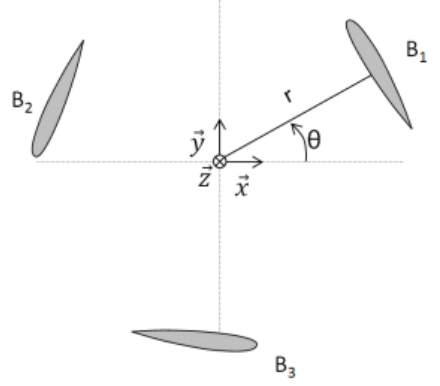

Fig 6. Parameterization of the turbine motion

Blade $\left(I_{l}\right)$ and generator inertia are taken into account for the computation, but blades arms inertia is assumed negligible. Generator inertia is split in two parts, $I_{2}$ and $I_{3}$, representing respectively to the stator and the rotor inertia. The coefficient 0.23 is the speed-reduction gear ratio between the rotor and the stator. $\ddot{\theta}$ denotes the acceleration of the turbine; $m$ and $r$ are the mass of a single blade and the radius of the turbine. $\overrightarrow{M_{f_{l / 0}}}$ is the moment resulting from the hydrodynamic forces acting on each blade related to point $\mathrm{O}$, origin of the coordinate system (fig. 6). The resistive torque of the generator $(\mathrm{Cr})$ is defined as the ratio between the mechanical power and the rotational velocity derived from the experimental test multiplied by a factor $f$.

The computation is 2 dimensions which means that there will be some differences in the forces computed with experiment as some elements of the turbine geometry are not represented (axe, support arms...) and 3 dimensions phenomenon are not taken into account. For each test a first run was performed with the resistive torque from the experiment $(f=1)$. If the velocity computed has not stabilized after 20 revolutions, $f$ was increased or reduced depending on the slope. This process was repeated further until the velocity reaches a value showing an acceptable difference with the experimental one.

Table II. - Numerical parameters

\begin{tabular}{|c|c|c|c|c|c|}
\hline Test & $\begin{array}{c}\boldsymbol{U}_{\boldsymbol{o}}(\mathbf{m} / \mathbf{s}) \\
\text { imposed } \\
\text { on the } \\
\text { inlet }\end{array}$ & $\begin{array}{c}\dot{\boldsymbol{\theta}}_{\boldsymbol{m}}(\mathrm{rad} / \mathbf{s}) \\
\text { used for the } \\
\text { initialization } \\
\text { case }\end{array}$ & $\Delta \mathbf{t}(\mathbf{s})$ & $\boldsymbol{\lambda}$ & $\boldsymbol{R} \boldsymbol{e}_{\boldsymbol{c}}$ \\
\hline $\mathbf{1}$ & 7.13 & 26.3 & $5.10^{-4}$ & 1.84 & $2.7 .10^{6}$ \\
\hline $\mathbf{2}$ & 9.9 & 44.6 & $3.10^{-4}$ & 2.25 & $4.7 .10^{6}$ \\
\hline $\mathbf{3}$ & 10.8 & 55.6 & $2.5 .10^{-4}$ & 2.57 & $5.38 .10^{6}$ \\
\hline
\end{tabular}

\section{Results and Discussion}

15 simulations, were performed to assess the optimum value of $f$ in each test. The latter represents the difference between the simulated and measured mechanical power. Figure 7 illustrates the behavior of the turbine in terms of rotational velocity to the changing values of $f$. For small values of $f$ (curves $\mathrm{A}$ to $\mathrm{C}$ ), the mean mechanical torque developed by the turbine is large in relation with the resistive torque. In consequence the turbine can accelerate and the mechanical torque decreases revolution after revolution to find an equilibrium with $\mathrm{Cr}$ in accordance with equation (1). On the contrary, for the greatest values of $f$, the resistive torque imposed remains higher to the mechanical torque so that the rotational velocity never stabilizes. The more important is the difference between the resistive and the simulated mechanical torque, the more significant is the acceleration or deceleration of the turbine.

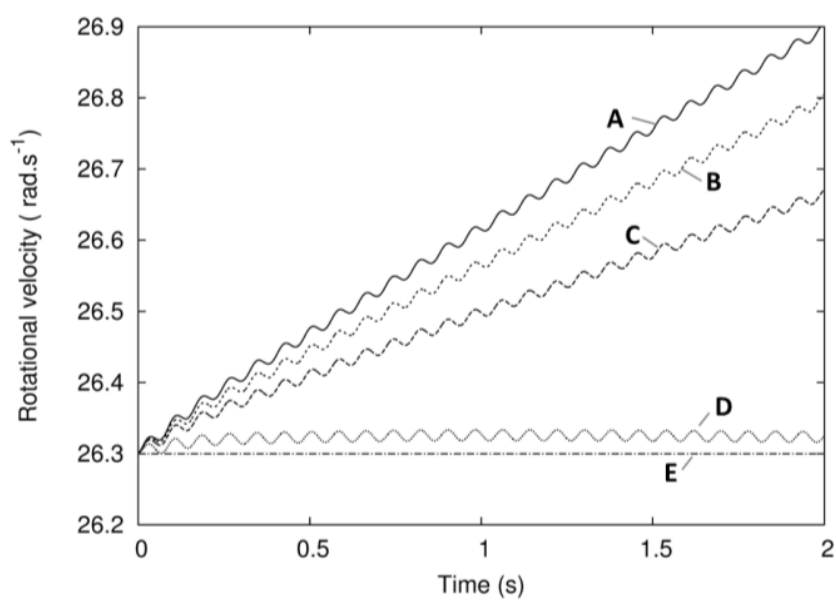

Fig 7. Evolution of the rotational velocity for increasing values of the resistive torque (A-D). The straight dash line (E) represents the target velocity corresponding to the experimental data.

By proceeding in the manner described in section 3, the optimal value of $f$ was determined for tests 1 to 3 (table III). Figure 8A shows the stabilized curves. The turbine reached a steady state between the $4^{\text {th }}$ and $6^{\text {th }}$ revolutions. The rotational speeds calculated are around $0.1 \%$ higher than the experimental one which is very close. This discrepancy is in the same order of magnitude than the uncertainties of the measurements realized during the experiment.

Once the resistive torque balances with the mechanical torque, the latter does not evolve further. It regulates to a mean constant value, the rotational speed follows the same behavior. However a slight deviation of the curve is observed.

The optimum values of $f$ are fairly close for the 3 tests. More tests should be investigated to obtain a clear tendency for $f$. It is not possible to say if it is possible to predict the complete behavior of the turbine with a fixed value of $f$ or if it should depend on the operating point. The tests correspond to different tip speed ratio $(\lambda)$ that may have an influence. Moreover, the form of the $\mathrm{Cp}$ curve for the various inlet velocities is not known; the position of the operating point on this curve may be a parameter to consider as it has been demonstrated for the 2 dimensions simulation of the power coefficient [9].

These results highlight the pulsating nature of the rotational velocity (Figure 8B). This is consistent with 
the theory on Darrieus rotor. As the blades rotate around the rotational axis, the angle of attack changes, this results in the variation of the hydrodynamic forces applied on the blades during a revolution. Two breaking zones and two acceleration zones can be identify inside the rotor. These oscillations have the same frequency than those of the mechanical torque, 3 oscillations per revolution which is in relation with the number of blades. The amplitude of these oscillations are very low (table III) and cannot modify critically the mechanical power in comparison to a constant rotational speed case. One can however wonder if the influence of these variations is still negligible in the fluid water with the density difference.

Table III. - Main parameters extract from the numerical results

\begin{tabular}{|c|c|c|c|c|}
\hline Test & $\boldsymbol{f}_{\text {op }}$ & $\begin{array}{c}\dot{\boldsymbol{\theta}}_{\text {calculated }} \\
\left(\text { (rad.s }^{-1}\right)\end{array}$ & $\begin{array}{c}\dot{\boldsymbol{\theta}}_{\text {target }} \\
\left(\text { (rad.s }^{-1}\right)\end{array}$ & oscillation \\
\hline $\mathbf{1}$ & 0.9 & 26.33 & 26,3 & $\pm 0,029 \%$ \\
\hline $\mathbf{2}$ & 0.5 & 44,66 & 44.6 & $\pm 0,0174 \%$ \\
\hline $\mathbf{3}$ & 0.5 & 55,63 & 55.6 & $\pm 0,0135 \%$ \\
\hline
\end{tabular}

To improve the evaluation of $f$, the experimental set-up has to be reviewed to perform the tests in more controlled conditions. Indeed a certain number of uncertainties exist mainly related to the flow pattern experience by the turbine. Indeed, the wind tunnel is open on the outside so the turbine may be influenced by external wind; The second fan situated on the right side of the main duct could have an effect. Recording the level of turbulence could dissipate some uncertainties. This may explain the difference in the optimal $f$ value found for the 3 tests.

The Cp-curve of the turbine should be determined numerically and experimentally for the fluid velocity considered. Different working conditions on these curves should be studied to investigate the sensibility of $f$ to $\lambda$ and to the position on the Cp-curve.

\section{Conclusion and Perspectives}

In this preliminary study, experimental tests were conducted on a vertical axis wind turbine loaded with a generator of which characteristics were determined. Numerical simulations were performed with Fluent (v14) in the same conditions with the mean of a User Defined Function developed to account for the fluid structure interaction of the turbine, as well as the resistive torque of a generator. The comparison of the two sets of data gave promising results and interesting clue to enhanced the method and assess $f$ in a more precise way in order to predict the behavior of a Darrieus turbine connected to a generator in 2 dimensions. The validity of the UDF function developed for this study has been demonstrated as the turbine reacts as expected (evolution of the rotational velocity and mechanical torque) to the various resistive torques imposed. Some improvement will be done in particular to correct the slight deviation of the rotational speed when the mechanical torque is stabilized. First, it concerns the order of the scheme used. The sensibility of the factor $f$ used in the 2 dimensions simulations to various parameters will also be investigated.
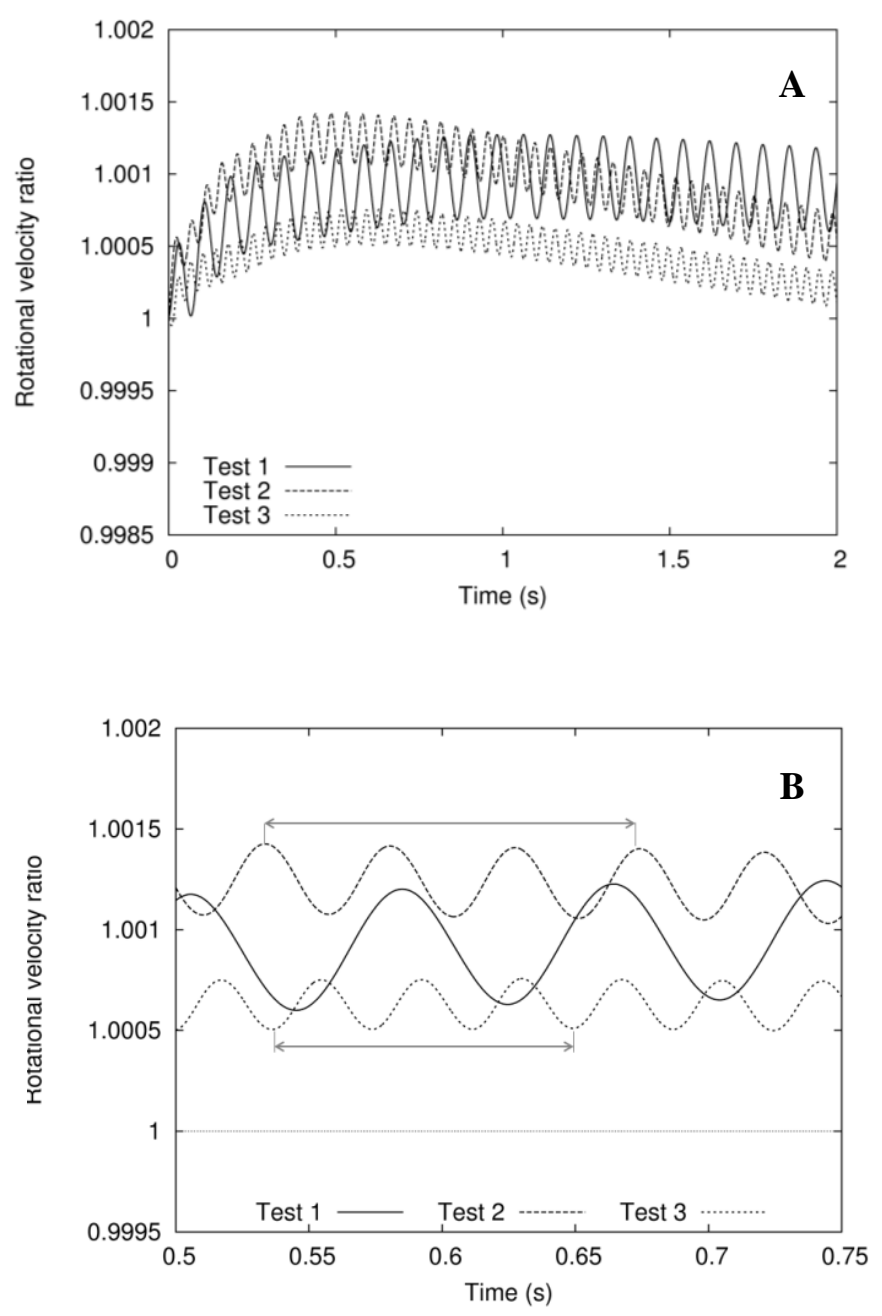

Fig 8 . Rotational velocity ratio $\dot{\theta}_{\text {calculated }} / \dot{\theta}_{\text {target }}$. The time lap corresponds to one revolution for test 1 . The arrows identify one revolution for test 2 and 3 .

\section{Acknowledgement}

This study was founded in association by the Low Normandy region and the society WIBEE (Wind Building Engineering). We would like to thank the Syndicat Mixte du Cotentin for financing the computational resources.

\section{References}

[1] L.S. Blunden, A.S. Bahaj, "Initial evaluation of tidal stream energy resources at Portland Bill, UK", Renewable Energy, 2006, Vol. 31, pp. 121-132.

[2] C.J. Simão Ferreira, "The near wake of the VAWT, 2D and 3D views of the VAWT aerodynamics",2009, PhD Thesis, Lisbon University.

[3] C.J. Simão Ferreira, H. Bijl, G. van Bussel, G. van Kuik, "Simulating Dynamic Stall in a 2D VAWT: Modeling strategy, verification and validation with Particle Image Velocity data", 2007, Journal of Physics: Conference Series 75, pp. 12-23 
[4] E. Amet, "Simulation numérique d'une hydrolienne à axe vertical de type Darrieus", 2009, PHD thesis, France, Grenoble, Polytechnica National Institute

[5] S. Lain, C. Osorio, "Simulation and Evaluation of a straight-bladed Darrieus-type cross flow flow marine turbine", Journal of Scientific \& Industrial Research, 2010, Vol. 69, pp. 906-912.

[6] J.H. Strickland, T. Smith, K. Sun, "A vortex model of the Darrieus turbine: An analytical and Experimental study", 1981, Sandia National Laboratoris, Albuquerque, NM, SAND81-7017

[7] G. Brochier, P. Fraunie, C. Beguier, I. Paraschivoiu "Water channel experiments of dynamic stall on Darrieus wind turbine blades", 1986, AIAA Journal of Propulsion and Power, Vol 2, pp. 445-449

[8] N. Fujisawa, S. Shibuya, "Observation of dynamic stall on Darrieus wind turbine blade", 2001, Journal of Wind Engineering and Industrial Aerodynamics, Vol. 89, pp. 201-214

[9] T. Maitre, E. Amet, C. Pellone, "Modeling of the flow in a Darrieus water turbine: Wall grid refinement analysis and comparison with experiments", 2013, Vol. 51, pp. 497-512

[10] L.J. Vermeer, J.N. Sørensen, A. Crespo, "Wind turbine wake aerodynamics", 2003, Progress in Aerospace Sciences, 2003, Vol. 39, pp. 467-510 